\title{
The supporting method for automatic diagnosis of prostatic hypertrophy
}

\author{
Wojciech Walendziuk ${ }^{1}$, Aleksander Sawicki ${ }^{2}$, Adam Idźkowski ${ }^{3}$ \\ $1,2,3$ Bialystok University of Technology, Poland \\ E-mails: ${ }^{1}$ w.walendziuk@pb.edu.pl (corresponding author), ${ }^{2}$ aleksander.sawicki.91@gmail.com, \\ 3 a.idzkowski@pb.edu.pl \\ (Received 29 March 2016; accepted 14 June 2016)
}

\begin{abstract}
In the paper numerical algorithms used in the automatic diagnosis of prostatic hypertrophy are presented. The liquid flow during urination was applied as a signal that describes the condition of prostate. In order to register the signal, the uroflowmeter was used. Patients were included in a two-step procedure. In the first step, an analysis of signal characteristics, such as maximum and the mean value with the use of Liverpool Nomogram, were performed. Then, the signal was tested for the presence of oscillation. For this purpose, an algorithm that generates the reference signal was created. Moreover, the similarity waveform was investigated with the help of the integral index. The diversity of signals indicated the presence of anomalies and had an impact on the final classification of the patient.
\end{abstract}

Keywords: uroflowmetry, urine flow, BPH diagnosis.

\section{Introduction}

The aim of this paper is to work out a numerical algorithm that realizes automatic classification of the urine flow speed characteristics. Such solution enables detecting oscillation in the urine flow, which may be a sign of the urinary tract disorders, e.g. benign prostatic hyperplasia (in the case of significant flow oscillations or the lack of the continuity of the characteristics). The methods which have been applied so far, e.g. Nomogram Liverpool, do not exploit information related to the urine flow characteristics. They are based on the analysis of the volume, average and maximum value of urination. The final diagnosis is formed by a doctor who analyses the shapes of time characteristics individually. The worked out algorithm will enable automate the screening tests process with the use of simple devices based on force transducers, such as uroflowmeters. They enable doing tests at a patient's home, and if the anomalies of the characteristics shape occur, the device will inform the user about the necessity of consulting the doctor. On the basis of the characteristics obtained from the machine, the doctor will be able to take decisions concerning further treatment. It should be stressed that the authors have never found any record of another solution concerning the urine flow curves classification with the use of a similar method. However, there are other solutions in terms of the urine flow charts identification based on neural networks [1].

\section{Algorithm of the dynamic urine flow analysis}

The urination is characterized by: the maximum and average flow rate, the urine volume, the time to maximum flow, the flow time and the time of urination [2-5]. Examples of the accurate and the impaired urination are shown in Fig. 1. 

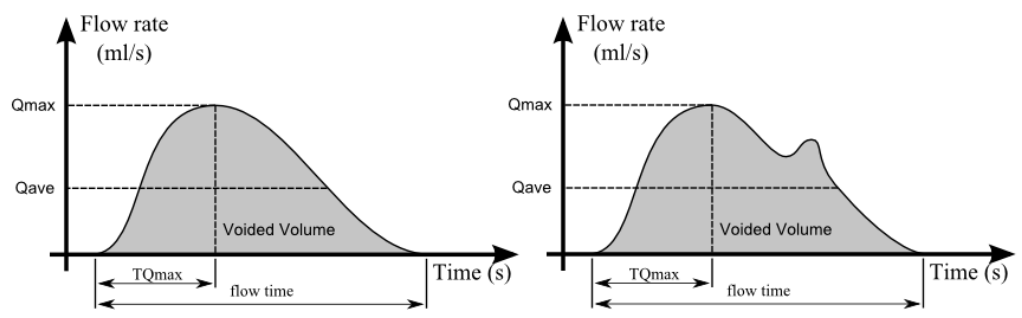

Fig. 1. The examples of urination patterns [6]

Nomograms considering the signal features such as maximum and average values are widely available in literature [7]. However, some anomalies of the flow (oscillations), not detected through the described parameters, can occur. They may indicate the occurrence of the disease and should be detected. In the further part of the study, a set of algorithms using nomograms, as well as the original idea for oscillations detection in the curve of the urination, are presented.

The described diagnostic algorithm worked in two steps. In the first one, the analysis of parameters such as the maximum and mean value, with the use of Liverpool Nomogram, was performed. The particular waveforms were replaced by the third and fourth order approximating polynomial and expressed by the following equation:

$\left\{\begin{array}{l}\text { Average }_{90 t h}=-1.87 \times 10^{-10} \times V_{v}^{4}+2.92 \times 10^{-7} \times V_{v}^{3}+0.072 \times V_{v}+4.656 \\ \text { Average }_{10 t h}=1.66 \times 10^{-8} \times V_{v}^{4}-1.77 \times 10^{-5} \times V_{v}^{2}+0.029 \times V_{v}+0.765\end{array}\right.$,

where: $V_{v}$ - voided volume.

If the determined average flow rate was between Average ${ }_{90 t h}$ and Average ${ }_{10 t h}$, the patient was considered to be within the normal range. An example of the nomogram is shown in Fig. 2.
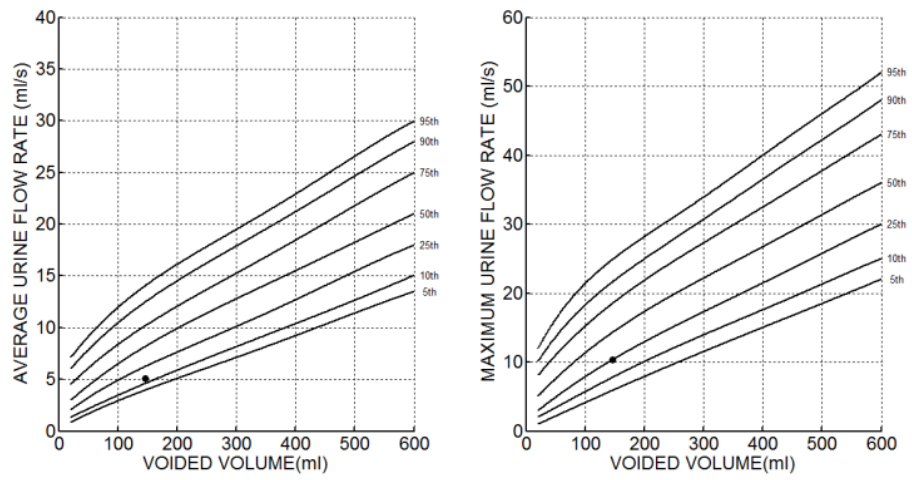

Fig. 2. Examples of generated nomograms

Then, a comparison between the generated reference signal with the urination signal was performed. The integral indicator described the similarity of signals and is expressed by the following equation:

$J=\int_{t_{0}}^{t_{k}}\left|Y-Y_{w}\right|$,

where: $t_{0}$ - the initial time, $t_{k}$ - the final time, $Y$ - signal value, $Y_{w}$ - value pattern. 
In order to generate the reference waveform, two third order Bézier curves [8] were used. The Bezier curve is generated dynamically for individual cases. It has the same time of achieving the maximum value, the same area under the curve, average and maximum value as the characteristics obtained during the urine flow tests.

The waveforms of the generated curves are presented in Fig. 3. On the left side, the Bézier curve is superimposed on the inaccurate waveform, while on the right side - the Bézier curve is within the normal range. For the first one, the coefficient value equalled $J=79.55$, while for the second one $-J=27.78$. The boundary coefficient value $J=70.00$ was empirically established as considered to be abnormal.

\section{Achieved results}

The application of the first of the described methods resulted in classifying 18 of the 50 available waveforms of urination within the normal range. However, in this group of waveforms, oscillations occurred in 8 cases. The second of the described methods was characterized by high numerical cost and was used in 18 selected waveforms of urination. Furthermore, in this case, the application of the algorithm enabled detection 6 of 8 irregularities.
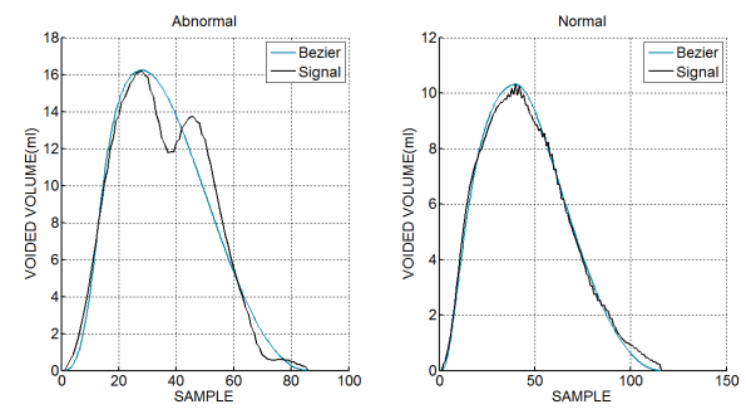

Fig. 3. The Bézier curves imposed on inaccurate and accurate waveforms

\section{Conclusions}

The aim of this paper was to present a method that can be applied in preliminary classification of the urine flow curves. It may be used in early diagnosis of e.g. prostatic hyperplasia. The worked out procedure of analyzing data from a uroflowmeter enables conducting an automatized classification of data that may indicate a urinary tract disorder.

It is worth underlying that application of the first method is insufficient for a definite diagnosis. In the group of patients considered to be healthy, waveforms with oscillations occurred as frequently as in 8 cases. Therefore, this method was not able to detect local anomalies. However, the application of the second method was characterized by high numerical cost, which considerably increased the efficiency of the algorithm in the detection of 6 inaccurate results out of 8 tests.

Undoubtedly, a diagnostic system should include at least two methods. In further study it is planned to replace the second method by a method using the wavelet transform.

\section{Acknowledgements}

The paper was prepared at Bialystok University of Technology within a framework of the S/WE/1/2013 and the MB/WE/4/2016 projects sponsored by Ministry of Science and Higher Education. 


\section{References}

[1] Altunay, S., et al. 2006. Interpretation of uroflow graphs with artificial neural networks, in Proceedings of IEEE Signal Processing and Communications Applications, 16-19 April 2006, Antalya, Turkey. Piscataway: IEEE. http://dx.doi.org/10.1109/SIU.2006.1659698

[2] Marks, L. S.; Roehrborn, C. G.; Andriole, G. L. 2006. Prevention of benign prostatic hyperplasia disease, Journal of Urology 176(4): 1299-1306. http://dx.doi.org/10.1016/j.juro.2006.06.022

[3] Abrams, P. 1997. Urodynamics. London: Springer-Verlag. 339 p. http://dx.doi.org/10.1007/978-1-4471-3598-2

[4] Chapple, C. R.; Steers, W. D. [eds.] 2011. Practical urology: essential principles and practice, London: Springer. 569 p. http://dx.doi.org/10.1007/978-1-84882-034-0

[5] Walendziuk, W.; Idzkowski, A. 2009. Portable acquisition system for domiciliary uroflowmetry, Journal of Vibroengineering 11(3): 592-596.

[6] Suryawanshi, A.; Joshi, A. 2012. Urine flow rate measurement based on volumetric pressure measurement principle, in Proceedings of 1st International Symposium on Physics and Technology of Sensors (ISPTS), March 7-10, 2012, Pune, India. Piscataway: IEEE, 334-337. http://dx.doi.org/10.1109/ispts.2012.6260961

[7] Haylen, B. T., et al. 1989. Maximum and average urine flow rates in normal male and female populations - the Liverpool nomograms, British Journal of Urology 64(1): 30-38. http://dx.doi.org/10.1111/j.1464-410X.1989.tb05518.x

[8] Prautzsch, H.; Boehm, W.; Paluszny, M. 2002. Bézier and B-spline techniques. Berlin Heidelberg: Springer-Verlag. 304 p. http://dx.doi.org/10.1007/978-3-662-04919-8 